\title{
Spatial Domain Adaptive Control of Nonlinear Rotary Systems Subject to Spatially Periodic Disturbances
}

\author{
Yen-Hsiu Yang ${ }^{1}$ and Cheng-Lun Chen ${ }^{2}$ \\ ${ }^{1}$ RED Division, Inventec Corporation, 66 Hou-Kang Street, Shin-Lin District, Taipei 111, Taiwan \\ ${ }^{2}$ Department of Electrical Engineering, National Chung Hsing University, Taichung 40227, Taiwan \\ Correspondence should be addressed to Cheng-Lun Chen, chenc@dragon.nchu.edu.tw
}

Received 22 February 2012; Revised 13 June 2012; Accepted 14 June 2012

Academic Editor: Baocang Ding

Copyright (C) 2012 Y.-H. Yang and C.-L. Chen. This is an open access article distributed under the Creative Commons Attribution License, which permits unrestricted use, distribution, and reproduction in any medium, provided the original work is properly cited.

\begin{abstract}
We propose a generic spatial domain control scheme for a class of nonlinear rotary systems of variable speeds and subject to spatially periodic disturbances. The nonlinear model of the rotary system in time domain is transformed into one in spatial domain employing a coordinate transformation with respect to angular displacement. Under the circumstances that measurement of the system states is not available, a nonlinear state observer is established for providing the estimated states. A two-degree-of-freedom spatial domain control configuration is then proposed to stabilize the system and improve the tracking performance. The first control module applies adaptive backstepping with projected parametric update and concentrates on robust stabilization of the closed-loop system. The second control module introduces an internal model of the periodic disturbances cascaded with a loop-shaping filter, which not only further reduces the tracking error but also improves parametric adaptation. The overall spatial domain output feedback adaptive control system is robust to model uncertainties and state estimated error and capable of rejecting spatially periodic disturbances under varying system speeds. Stability proof of the overall system is given. A design example with simulation demonstrates the applicability of the proposed design.
\end{abstract}

\section{Introduction}

Rotary systems play important roles in various industry applications, for example, packaging, printing, assembly, fabrication, semiconductor, robotics, and so forth. Design of control algorithm for a motion system often comes up with nonlinearities and uncertainties. Nonlinearities are either inherent to the system or due to the dynamics of actuators and sensors. Uncertainties are mainly caused by unmodeled dynamics, parametric uncertainty, and disturbances. For dealing with nonlinearities, common techniques, for example, feedback 
linearization and backstepping, are to utilize feedback to cancel all or part of the nonlinear terms. On the other hand, design techniques for conducting disturbance rejection or attenuation in control systems mostly originate from the internal model principle [1], for example, those incorporating or estimating the exosystem of the disturbances [2-6]. Conventional controllers are mostly time-based controllers as they are synthesized and operate in temporal or time domain. Several researches [7-9] have started studying spatial domain controllers ever since a repetitive controller design was initiated by Nakano et al. [10]. In the design of Nakano et al., the repetitive control system has its repetitive kernel (i.e., $e^{-L s}$ with positive feedback) synthesized and operated with respect to a spatial coordinate, for example, angular position or displacement. Hence its capability for rejecting or tracking spatially periodic disturbances or references will not degrade when the controlled system operates at varying speed. All existing studies propose design methods starting with a linear time-invariant (LTI) system. After reformulation, a nonlinear open-loop system is obtained in spatial domain. Subsequently, the open-loop system is either linearized around an operating speed or regarded as a quasi-linear parameter varying (quasi-LPV) system and then adjoined with the spatial domain internal model of the tracking or disturbance signal. Design paradigms based on linear (robust) control theory are then applied to the resulting augmented system. However, presuming the open-loop system to be LTI and resorting to design paradigm of linear control will inevitably restrict the applicability and limit the achievable performance of a design method. Chen and Yang [11] introduced a new spatial domain control scheme based on a second-order LTI system with availability of state measurements. To achieve robust stabilization and high-performance tracking, a two-module control configuration is constructed. One of the modules utilizes adaptive backstepping with projected parametric adaptation to robustly stabilize the system. The other module incorporates a spatial domain internal model of the disturbances cascaded with a loopshaping filter to improve the tracking performance.

This paper extends the work of Chen and Yang [11,12]. The control scheme has been generalized such that it is applicable to a class of nonlinear systems (instead of just LTI systems). Moreover, the major shortcoming in Chen and Yang's design [11], that is, which requires full-state feedback, is resolved by incorporation of a nonlinear state observer. Various types of nonlinear state observers have been developed and put into use in the past (e.g., $[13,14])$. This paper will study the feasibility of incorporating a $K$-filter-type state observer [13] into the proposed design. The proposed system incorporating the state observer can be proved to be stable under bounded disturbance and system uncertainties. An illustrative example is given for demonstration and derivation of the control algorithm. Simulation is performed to verify the feasibility and effectiveness of the proposed scheme. Compared to the preliminary work in [12] (which is only applicable to second order systems), the results have been generalized to be suited for $n$th order systems. Specifically, the design and stability proof are more comprehensive and rigorous than those presented in [12].

Recently, there have been emerging design techniques based on adaptive fuzzy control (AFC), which may cope with nonlinearities and uncertainties with unknown structures [15-17]. The major differences between those techniques and the proposed one are as follows: (1) design being time based (AFC) versus spatial based (the proposed approach); (2) assuming less information about the nonlinearities/uncertainties (AFC) versus more information about the nonlinearities/uncertainties (the proposed approach). Note that the spatial-based design is not just a change of the independent variable from time to angular displacement. A nonlinear coordinate transformation is actually involved. Therefore, the systems under consideration in AFC and the proposed method are different. Next, 
the capability of the design approaches suggested in AFC for tackling systems subject to a more generic class of nonlinearities/uncertainties lies in the usage of a fuzzy system to approximate those nonlinearities/uncertainties. It is actually not clear regarding the complexity of the fuzzy system (i.e., number of membership functions) that should be used to achieve the required control performance. It is also not clear whether or not the control effort is reasonable. In general, when characteristics of the uncertainties or disturbances are known, such information should be incorporated as much as possible into the design to enhance performance, avoid conservativeness, and result in sensible control input. Hence, instead of assuming the disturbances to be generic (probably just being bounded as by AFC), the proposed design is aiming at a type of disturbances specific to rotary systems and utilizes the spatially periodic nature of the disturbances to establish a well-defined control module integrated into the overall control configuration.

This paper is organized as follows. Reformulation of a generic nonlinear rotary system with respect to angular displacement will be presented in Section 2. Design of the state observer is described in Section 3. Section 4 will cover derivation and stability analysis of the proposed spatial domain output feedback control scheme. Simulation verification for the proposed scheme will be presented in Section 5. Conclusion is given in Section 6.

\section{Problem Formulation}

In this section, we show how a generic NTI model can be transformed into an NPI model by choosing an alternate independent variable (angular displacement instead of time) and defining a new set of states (or coordinates) with respect to the angular displacement. Note that the transformation described here is equivalent to a nonlinear coordinate transformation or a diffeomorphism. The NPI model will be used for the subsequent design and discussion,

$$
\begin{gathered}
\dot{x}(t)=\left[f_{t}\left(x(t), \phi_{f}\right)+\Delta f_{t}\left(x(t), \phi_{f}\right)\right]+\left[g_{t}\left(x(t), \phi_{g}\right)+\Delta g_{t}\left(x(t), \phi_{g}\right)\right] u(t), \\
y=\Psi x(t)+d_{y}(t)=x_{1}(t)+d_{y}(t),
\end{gathered}
$$

where $x(t)=\left[\begin{array}{lll}x_{1}(t) & \cdots & x_{n}(t)\end{array}\right]^{T}, \Psi=\left[\begin{array}{llll}1 & 0 & \cdots & 0\end{array}\right]$, and $u(t)$ and $y(t)$ correspond to control input and measured output angular velocity of the system, respectively. $d_{y}(t)$ represents a class of bounded output disturbances which constitutes (dominant) spatially periodic and band-limited (or nonperiodic) components. Here we refer band-limited disturbances to signals whose Fourier transform or power spectral density is zero above a certain finite frequency. The only available information of the disturbances is the number of distinctive spatial frequencies and the spectrum distribution for band-limited disturbance components. $f_{t}\left(x(t), \phi_{f}\right)$ and $g_{t}\left(x(t), \phi_{g}\right)$ are known vector-valued functions with unknown but bounded system parameters, that is, $\phi_{f}=\left[\begin{array}{lll}\phi_{f 1} & \cdots & \phi_{f k}\end{array}\right]$ and $\phi_{g}=\left[\begin{array}{lll}\phi_{g 1} & \cdots & \phi_{g l}\end{array}\right] ; \Delta f_{t}\left(x(t), \phi_{f}\right)$ and $\Delta g_{t}\left(x(t), \phi_{g}\right)$ represent unstructured modeling inaccuracy, which are also assumed to be bounded. Instead of using time $t$ as the independent variable, consider an alternate independent variable $\theta=\lambda(t)$, that is, the angular displacement. Since by definition

$$
\lambda(t)=\int_{0}^{t} \omega(\tau) d \tau+\lambda(0)
$$


where $\omega(t)$ is the angular velocity, the following condition:

$$
\omega(t)=\frac{d \theta}{d t}>0, \quad \forall t>0,
$$

will guarantee that $\lambda(t)$ is strictly monotonic such that $t=\lambda^{-1}(\theta)$ exists. Thus all the variables in the time domain can be transformed into their counterparts in the $\theta$-domain, that is,

$$
\begin{gathered}
\widehat{x}(\theta)=x\left(\lambda^{-1}(\theta)\right), \quad \widehat{y}(\theta)=y\left(\lambda^{-1}(\theta)\right), \\
\widehat{u}(\theta)=u\left(\lambda^{-1}(\theta)\right), \quad \widehat{d}(\theta)=d\left(\lambda^{-1}(\theta)\right), \\
\widehat{\omega}(\theta)=\omega\left(\lambda^{-1}(\theta)\right),
\end{gathered}
$$

where we denote $\hat{\bullet}$ as the $\theta$-domain representation of $\bullet$. Note that, in practice, (2.3) can usually be satisfied for most rotary systems where the rotary component rotates only in one direction. Since

$$
\frac{d x(t)}{d t}=\frac{d \theta}{d t} \frac{d \widehat{x}(\theta)}{d \theta}=\widehat{\omega}(\theta) \frac{d \widehat{x}(\theta)}{d \theta}
$$

Equation (2.1) can be rewritten as

$$
\begin{gathered}
\widehat{\omega}(\theta) \frac{d \widehat{x}(\theta)}{d \theta}=\left[f_{t}\left(\widehat{x}(\theta), \phi_{f}\right)+\Delta f_{t}\left(\widehat{x}(\theta), \phi_{f}\right)\right]+\left[g_{t}\left(\widehat{x}(\theta), \phi_{g}\right)+\Delta g_{t}\left(\widehat{x}(\theta), \phi_{g}\right)\right] \widehat{u}(\theta), \\
\widehat{y}(\theta)=\Psi \widehat{x}(\theta)+\widehat{d}_{y}(\theta)=\widehat{x}_{1}(\theta)+\widehat{d}_{y}(\theta) .
\end{gathered}
$$

Equation (2.6) can be viewed as a nonlinear position-invariant (as opposed to the definition of time-invariant) system with the angular displacement $\theta$ as the independent variable. Note that the concept of transfer function is still valid for linear position-invariant systems if we define the Laplace transform of a signal $\widehat{g}(\theta)$ in the angular displacement domain as

$$
\widehat{G}(\widetilde{s})=\int_{0}^{\infty} \widehat{g}(\theta) e^{-\tilde{s} \theta} d \theta
$$

This definition will be useful for describing the linear portion of the overall control system.

\section{Nonlinear State Observer}

Drop the $\theta$ notation and note that (2.6) can be expressed as a standard nonlinear system:

$$
\dot{\hat{x}}=f\left(\widehat{x}, \phi_{f}\right)+g\left(\widehat{x}, \phi_{g}\right) \widehat{u}+\widehat{d}_{s}, \quad \widehat{y}=h(\widehat{x})+\widehat{d}_{y}=\widehat{\omega}+\widehat{d}_{y}
$$


where terms involving unstructured uncertainty are merged into $\widehat{d}_{s}=\Delta f\left(\widehat{x}, \phi_{f}\right)+$ $\Delta g\left(\widehat{x}, \phi_{g}\right) \widehat{u}$ with

$$
\Delta f\left(\widehat{x}, \phi_{f}\right)=\frac{\Delta f_{t}\left(\widehat{x}, \phi_{f}\right)}{\widehat{x}_{1}}, \quad \Delta g\left(\widehat{x}, \phi_{g}\right)=\frac{\Delta g_{t}\left(\widehat{x}, \phi_{g}\right)}{\widehat{x}_{1}}
$$

In addition, we have

$$
f\left(\widehat{x}, \phi_{f}\right)=\frac{f_{t}\left(\widehat{x}, \phi_{f}\right)}{\widehat{x}_{1}}, \quad g\left(\widehat{x}, \phi_{g}\right)=\frac{g_{t}\left(\widehat{x}, \phi_{g}\right)}{\widehat{x}_{1}}, \quad h(\widehat{x})=\widehat{\omega}=\widehat{x}_{1} .
$$

The state variables have been specified such that the angular velocity $\widehat{\omega}$ is equal to $\widehat{x}_{1}$, that is, the undisturbed output $h(\widehat{x})$. It is not difficult to verify that (3.1) has the same relative degree in $D_{0}=\left\{\widehat{x} \in \mathbb{R}^{n} \mid \widehat{x}_{1} \neq 0\right\}$ as the NTI model in (2.1). If (3.1) has relative degree $r$, we can define the following nonlinear coordinate transformation:

$$
\widehat{z}=T(\widehat{x})=\left[\begin{array}{c}
\psi_{1}(\widehat{x}) \\
\vdots \\
\frac{\psi_{n-r}(\widehat{x})}{h(\widehat{x})} \\
\vdots \\
L_{f}^{r-1} h(\widehat{x})
\end{array}\right] \triangleq\left[\frac{\widehat{z}_{2}}{\widehat{z}_{1}}\right]
$$

where $\psi_{1}$ to $\psi_{n-r}$ are chosen such that $T(\widehat{x})$ is a diffeomorphism on $D_{0} \subset D$ and

$$
L_{g} \psi_{i}(\widehat{x})=0, \quad 1 \leq i \leq n-r, \quad \forall \widehat{x} \in D_{0} .
$$

With respect to the new coordinates, that is, $\widehat{z}_{1}$ and $\widehat{z}_{2}$, (3.1) can be transformed into the socalled normal form, that is,

$$
\begin{gathered}
\dot{\vec{z}}_{2}=\left.L_{f} \psi(\widehat{x})\right|_{\widehat{x}=T^{-1}(\hat{z})}+\widehat{d}_{s o} \triangleq \Psi\left(\widehat{z}_{1}, \widehat{z}_{2}\right) \\
\dot{\bar{z}}_{1}=A_{c} \widehat{z}_{1}+B_{c}\left[\left.L_{g} L_{f}^{r-1} h(\widehat{x})\right|_{\widehat{x}=T^{-1}(\hat{z})}\right]\left[\widehat{u}+\left.\frac{L_{f}^{r} h(\widehat{x})}{L_{g} L_{f}^{r-1} h(\widehat{x})}\right|_{\widehat{x}=T^{-1}(\widehat{z})}\right]+\widehat{d}_{s i} \\
\widehat{y}=C_{c} \widehat{z}_{1}+\widehat{d}_{y}
\end{gathered}
$$

where $\widehat{d}_{s o}$ and $\widehat{d}_{s i}=\left[\begin{array}{lll}\hat{d}_{s i_{1}} & \cdots & \widehat{d}_{s i_{r}}\end{array}\right]^{T}$ come from $\widehat{d}_{s}$ going through the indicated coordinate transformation. $\widehat{z}_{1}=\left[\begin{array}{lll}\hat{z}_{11} & \cdots & \widehat{z}_{1 r}\end{array}\right] \in \mathbb{R}^{r}, \widehat{z}_{2} \in \mathbb{R}^{n-r}$, and $\left(A_{c}, B_{c}, C_{c}\right)$ is a canonical form representation of a chain of $r$ integrators. The first equation is called internal dynamics and the second is called external dynamics. Internal dynamics which is not affected by the control $u$. By setting $\widehat{z}_{1}=0$ in that equation, we obtain

$$
\dot{\hat{z}}_{2}=\Psi\left(0, \widehat{z}_{2}\right),
$$


which is the zero dynamics of (3.1) or (3.6). The system is called minimum phase if (3.7) has an asymptotically stable equilibrium point in the domain of interest. To allow us to present the proposed algorithm and stability analysis in a simpler context, we will make the following assumptions for the subsequent derivation:

(1) $f\left(\widehat{x}(\theta), \phi_{f}\right)$ and $g\left(\widehat{x}(\theta), \phi_{g}\right)$ are linearly related to those unknown system parameters, that is,

$$
\begin{aligned}
& f\left(\widehat{x}(\theta), \phi_{f}\right)=\phi_{1} f_{1}(\widehat{x}(\theta))+\cdots+\phi_{f k} f_{k}(\widehat{x}(\theta)) \\
& g\left(\widehat{x}(\theta), \phi_{g}\right)=\phi_{g 1} g_{1}(\widehat{x}(\theta))+\cdots+\phi_{g l} g_{l}(\widehat{x}(\theta)) .
\end{aligned}
$$

(2) Equation (3.1) is minimum phase, and the internal dynamics in (3.6) is ISS (inputto-state stable).

(3) The output disturbance is sufficiently smooth, that is, $\hat{\vec{d}}_{y}, \ldots, \widehat{d}_{y}^{(r)}$ exist,

(4) $\widehat{d}_{s i_{1}}^{(r-1)}, \widehat{d}_{s i_{2}}^{(r-2)}, \ldots, \dot{\hat{d}}_{s i_{r-1}}$ exist, that is, the transformed unstructured uncertainty is sufficiently smooth.

(5) The reference command $\widehat{y}_{m}$ and its first $r$ derivates are known and bounded. Moreover, the signal $\widehat{y}_{m}^{(r)}$ is piecewise continuous.

With assumption (2), we focus on designing a nonlinear state observer for external dynamics of (3.6),

$$
\dot{\bar{z}}_{1}=A_{c} \widehat{z}_{1}+B_{c}\left[\left.L_{g} L_{f}^{r-1} h(\widehat{x})\right|_{\widehat{x}=T^{-1}(\hat{z})}\right]\left[\widehat{u}+\left.\frac{L_{f}^{r} h(\widehat{x})}{L_{g} L_{f}^{r-1} h(\widehat{x})}\right|_{\widehat{x}=T^{-1}(\hat{z})}\right]+\widehat{d}_{s i}
$$

Since $f(\widehat{x})$ and $g(\widehat{x})$ are linearly related to system parameters, $L_{g} L_{f}^{r-1} h(\widehat{x})$ and $L_{g} L_{f}^{r-1} h(\widehat{x})$ can be written as $L_{f}^{r} h(\widehat{x})=\Theta^{T} W_{f}(\widehat{x})$ and $L_{g} L_{f}^{r-1} h(\widehat{x})=\Theta^{T} W_{g}(\widehat{x})$, where $W_{f}(\widehat{x})$ and $W_{g}(\widehat{x})$ are two nonlinear functions, and $\Theta=\left[\begin{array}{llllllll}\phi_{f 1} & \cdots & \phi_{f k} & \phi_{g 1} & \cdots & \phi_{g l} & \cdots\end{array}\right]^{T}=\left[\begin{array}{llll}\phi_{1} & \cdots & \phi_{\ell}\end{array}\right]^{T} \in \mathbb{R}^{\ell}$, where $\ell$ is the number of unknown parameters. Next, we adopt the following observer structure: $\dot{\bar{z}}_{1}=A_{0} \bar{z}_{1}+\bar{k} y+F(y, u)^{T} \Theta$, where $\bar{z}_{1}=\left[\begin{array}{lll}\bar{z}_{11} & \cdots & \bar{z}_{1 r}\end{array}\right]^{T}$ is the estimate of $z_{1}$, and $\bar{W}_{f}(y)$ and $\bar{W}_{g}(y)$ are nonlinear functions with the same structure as $W_{f}(x)$ and $W_{g}(x)$ except that each entry of $x$ is replaced by $y$. Furthermore,

$$
A_{0}=\left[\begin{array}{cc}
-k_{1} & \\
\vdots & I_{(r-1) \times(r-1)} \\
-k_{r} & 0_{1 \times(r-1)}
\end{array}\right], \quad \bar{k}=\left[\begin{array}{lll}
k_{1} & \cdots & k_{r}
\end{array}\right]^{T}, \quad F(y, u)^{T}=\left[\begin{array}{c}
0_{(r-1) \times \ell} \\
\bar{W}_{f}^{T}(y)+\bar{W}_{g}^{T}(y) u
\end{array}\right] \in \mathbb{R}^{r \times \ell}
$$

By properly choosing $\bar{k}$, the matrix $A_{0}$ can be made Hurwitz. Define the state estimated error as $\varepsilon \triangleq\left[\begin{array}{lll}\varepsilon_{z_{11}} & \cdots & \varepsilon_{z_{1 r}}\end{array}\right]^{T} \triangleq z_{1}-\bar{z}_{1}$. The dynamics of the estimated error can be obtained as $\dot{\varepsilon}=A_{0} \varepsilon+\Delta$, where $\Delta=-\bar{k} d_{y}+B_{c} \Theta^{T}\left[W_{g}(x)-\bar{W}_{g}(y)\right] u+B_{c} \Theta^{T}\left[W_{f}(x)-\bar{W}_{f}(y)\right]+d_{s i}$. To 
proceed, the role of the state observer is replaced by $\bar{z}_{1} \triangleq \xi+\Omega^{T} \Theta$ and the following two K-filters:

$$
\dot{\xi}=A_{0} \xi+\bar{k} y, \quad \dot{\Omega}^{T}=A_{0} \Omega^{T}+F(y, u)^{T},
$$

such that $\xi=\left[\begin{array}{lll}\xi_{11} & \cdots & \xi_{1 r}\end{array}\right]^{T} \in \mathbb{R}^{r}$ and $\Omega^{T} \triangleq\left[\begin{array}{lll}v_{1} & \cdots & v_{\ell}\end{array}\right] \in \mathbb{R}^{r \times \ell}$. Decompose the second equation of (3.11) into $\dot{v}_{j}=A_{0} v_{j}+e_{r} \sigma_{j}, j=1,2, \ldots, \ell$, where $e_{r}=\left[\begin{array}{llll}0 & \cdots & 0 & 1\end{array}\right] \in \mathbb{R}^{r}$ and

$\sigma_{j}=w_{1 j}+w_{2 j} u$ with $w_{1 j}$ and $w_{2 j}$ are the $j$ th columns of $\bar{W}_{f}^{T}(y)$ and $\bar{W}_{g}^{T}(y)$, respectively. With the definition of the state estimated error $\varepsilon$, the state estimate $\bar{z}_{1}$, and (3.11), we acquire the following set of equations which will be used in the subsequent design:

$$
z_{1 k}=\bar{z}_{1 k}+\varepsilon_{z_{1 k}}=\xi_{1 k}+\sum_{j=1}^{\ell} v_{j, k} \phi_{j}+\varepsilon_{z_{1 k}}, \quad k=1, \ldots, r,
$$

where $\bullet_{j, i}$ denotes the $i$ th row of $\bullet_{j}$.

\section{Spatial Domain Output Feedback Adaptive Control System}

To apply adaptive backstepping method, we firstly rewrite the derivative of output $\hat{y}$ as

$$
\dot{\hat{y}}=\dot{\hat{z}}_{11}+\dot{\hat{d}}_{y}=\widehat{z}_{12}+\widehat{d}_{s i_{1}}+\dot{\hat{d}}_{y}=\bar{z}_{12}+\varepsilon_{\hat{z}_{12}}+\widehat{d}_{s i_{1}}+\dot{\hat{d}}_{y}
$$

With the second equation in (3.12), (4.1) can be written as

$$
\dot{\hat{y}}=\bar{z}_{12}+\varepsilon_{\widehat{z}_{12}}+\widehat{d}_{s i_{1}}+\dot{\hat{d}}_{y}=\xi_{12}+v_{\ell, 2} \phi_{\ell}+\bar{\omega}^{T} \Theta+\varepsilon_{\hat{z}_{12}}+\widehat{d}_{s i_{1}}+\dot{\hat{d}}_{y}
$$

where $\bar{\omega}^{T}=\left[\begin{array}{llll}v_{1,2} & \cdots & v_{\ell-1,2} & 0\end{array}\right]$.

In view of designing output feedback backstepping with $K$-filters, we need to find a set of $K$-filter parameters, that is, $v_{\ell, 2}, \ldots, v_{1,2}$, separated from $\hat{u}$ by the same number of integrators between $\widehat{z}_{12}$ and $\widehat{u}$. From (3.11), we can see that $v_{\ell, 2}, \ldots, v_{1,2}$ are all candidates if $w_{2 j}$ are not zero. In the following derivation, we assume that $v_{\ell, 2}$ is selected. Hence, the system incorporating the $K$-filters can be expressed as

$$
\begin{gathered}
\dot{\hat{y}}=\xi_{12}+v_{\ell, 2} \phi_{\ell}+\bar{\omega}^{T} \Theta+\varepsilon_{\bar{z}_{12}}+\widehat{d}_{s i_{1}}+\dot{\hat{d}}_{y}, \quad \dot{v}_{\ell, i}=v_{\ell, i+1}-k_{i} v_{\ell, 1}, \quad i=2, \ldots, r-1, \\
\dot{v}_{\ell, r}=-k_{r} v_{\ell, 1}+w_{1 \ell}+w_{2 \ell} \widehat{u} .
\end{gathered}
$$

To apply adaptive backstepping to (4.3), a new set of coordinates will be introduced

$$
z_{1}=\widehat{y}-\widehat{y}_{m}, \quad z_{i}=v_{\ell, i}-\alpha_{i-1}, \quad i=2, \ldots, r,
$$


where $\widehat{y}_{m}$ is the prespecified reference command and $\alpha_{i-1}$ is the virtual input which will be used to stabilize each state equation. For simplicity, we define $\partial \alpha_{0} / \partial \widehat{y} \triangleq-1$ for subsequent derivations.

Step $1(i=1)$. With $(4.4)$, the first state equation of $(4.3)$ can be expressed as

$$
\dot{z}_{1}=\xi_{12}+z_{2} \phi_{\ell}+\alpha_{1} \phi_{\ell}+\bar{\omega}^{T} \Theta+\varepsilon_{\widehat{z}_{12}}+\widehat{d}_{s i_{1}}+\dot{\hat{d}}_{y}-\dot{\hat{y}}_{m}
$$

Consider a Lyapunov function $V_{1}=(1 / 2) z_{1}^{2}$ and calculate its derivative

$$
\dot{V}_{1}=z_{1} \dot{z}_{1}=z_{1}\left(\xi_{12}+z_{2} \phi_{\ell}+\alpha_{1} \phi_{\ell}+\bar{\omega}^{T} \Theta+\varepsilon_{\widehat{z}_{12}}+\widehat{d}_{s i_{1}}+\dot{\hat{d}}_{y}-\dot{\hat{y}}_{m}\right)
$$

Define the estimates of $\phi_{i}$ as $\tilde{\phi}_{i}$ and $\Phi=\left[\begin{array}{lll}\Phi_{1} & \cdots & \Phi_{\ell}\end{array}\right]=\Theta-\tilde{\Theta}$, where $\tilde{\Theta}=$ $\left[\begin{array}{lllllll}\tilde{\phi}_{f 1} & \cdots & \tilde{\phi}_{f k} & \tilde{\phi}_{g 1} & \cdots & \tilde{\phi}_{g l} & \cdots\end{array}\right]^{T}=\left[\begin{array}{lll}\tilde{\phi}_{1} & \cdots & \tilde{\phi}_{\ell}\end{array}\right]^{T} \in \mathbb{R}^{\ell}$. Note that $\Theta$ is the "true" parameter vector while $\tilde{\Theta}$ is the estimated parameter vector. Design the virtual input $\alpha_{1}$ as $\alpha_{1}=\bar{\alpha}_{1} / \tilde{\phi}_{\ell}$ and specify

$$
\begin{aligned}
\bar{\alpha}_{1} & =\frac{1}{z_{1}}\left(-z_{1} \xi_{12}-z_{1} z_{2} \tilde{\phi}_{\ell}-z_{1} \bar{\omega} \tilde{\Theta}+z_{1} \dot{\hat{y}}_{m}-c_{1} z_{1}^{2}-d_{1} z_{1}^{2}-g_{1} z_{1}^{2}\right) \\
& =-\xi_{12}-z_{2} \tilde{\phi}_{\ell}-\bar{\omega}^{T} \widetilde{\Theta} \dot{\hat{y}}_{m}-c_{1} z_{1}-d_{1} z_{1}-g_{1} z_{1}
\end{aligned}
$$

where $c_{i}, d_{i}, g_{i}$ are variables. Therefore, (4.6) becomes

$$
\dot{V}_{1}=-c_{1} z_{1}^{2}-d_{1} z_{1}^{2}-g_{1} z_{1}^{2}+\tau_{1} \Phi+z_{1}\left(\varepsilon_{\hat{z}_{12}}+\widehat{d}_{s i_{1}}+\dot{d}_{y}\right)
$$

where $\tau_{1} \Phi=z_{1} z_{2} \Phi_{\ell}+\alpha_{1} \Phi_{\ell}+z_{1} \bar{\omega}^{T} \Phi$.

Step $2(i=2, \ldots, r-1)$. With respect to the new set of coordinates (4.4), the second equation of (4.3) can be rewritten as

$$
\begin{aligned}
\dot{z}_{i}=z_{i+1}+\alpha_{i}-k_{i} v_{\ell, 1}- & {\left[\frac{\partial \alpha_{i-1}}{\partial \hat{y}}\left(\xi_{12}+v_{\ell, 2} \phi_{\ell}+\bar{\omega}^{T} \Theta+\varepsilon_{\widehat{z}_{12}}+\widehat{d}_{s i_{1}}+\dot{\hat{d}}_{y}\right)+\frac{\partial \alpha_{i-1}}{\partial \xi}\left(A_{0} \xi+\bar{k} \hat{y}\right)\right.} \\
+ & \left.\frac{\partial \alpha_{i-1}}{\partial \tilde{\Theta}} \dot{\widetilde{\Theta}} \sum_{j=1}^{\ell} \frac{\partial \alpha_{i-1}}{\partial v_{j}}\left(A_{0} v_{j}+e_{r} \sigma_{j}\right)+\sum_{j=1}^{i-1} \frac{\partial \alpha_{i-1}}{\partial \widehat{y}_{m}^{(j-1)}} \widehat{y}_{m}^{(j)}\right] .
\end{aligned}
$$


Consider a Lyapunov function $V_{i}=\sum_{j=1}^{i-1} V_{j}+(1 / 2) z_{i}^{2}$ and its derivative

$$
\begin{aligned}
& \dot{V}_{i}=\sum_{j=1}^{i-1} \dot{V}_{j}+z_{i}\left\{z_{i+1}+\alpha_{i}-k_{i} v_{\ell, 1}-\right. {\left[\frac{\partial \alpha_{i-1}}{\partial \widehat{y}}\left(\xi_{12}+v_{\ell, 2} \phi_{\ell}+\bar{\omega}^{T} \Theta+\varepsilon_{\widehat{z}_{12}}+\widehat{d}_{s i_{1}}+\dot{\hat{d}}_{y}\right)\right.} \\
&+ \frac{\partial \alpha_{i-1}}{\partial \xi}\left(A_{0} \xi+\bar{k} \hat{y}\right)+\frac{\partial \alpha_{i-1}}{\partial \widetilde{\Theta}} \dot{\tilde{\Theta}} \sum_{j=1}^{\ell} \frac{\partial \alpha_{i-1}}{\partial v_{j}}\left(A_{0} v_{j}+e_{r} \sigma_{j}\right) \\
&\left.\left.+\sum_{j=1}^{i-1} \frac{\partial \alpha_{i-1}}{\partial \widehat{y}_{m}^{(j-1)}} \widehat{y}_{m}^{(j)}\right]\right\}
\end{aligned}
$$

Specify

$$
\begin{aligned}
\alpha_{i}=\frac{1}{z_{i}}\left\{-z_{i} z_{i+1}+z_{i} k_{i} v_{\ell, 1}+z_{i}\right. & {\left[\frac{\partial \alpha_{i-1}}{\partial \widehat{y}}\left(\xi_{12}+v_{\ell, 2} \tilde{\phi}_{\ell}+\bar{\omega}^{T} \widetilde{\Theta}\right)+\frac{\partial \alpha_{i-1}}{\partial \xi}\left(A_{0} \xi+\bar{k} \hat{y}\right)\right.} \\
& \left.+\frac{\partial \alpha_{i-1}}{\partial \widetilde{\Theta}} \dot{\tilde{\Theta}} \sum_{j=1}^{\ell} \frac{\partial \alpha_{i-1}}{\partial v_{j}}\left(A_{0} v_{j}+e_{r} \sigma_{j}\right)+\sum_{j=1}^{i-1} \frac{\partial \alpha_{i-1}}{\partial \widehat{y}_{m}^{(j-1)}} \widehat{y}_{m}^{(j)}\right] \\
& \left.-c_{i} z_{i}^{2}-d_{i}\left(\frac{\partial \alpha_{i-1}}{\partial \widehat{y}}\right)^{2} z_{i}^{2}-g_{i}\left(\frac{\partial \alpha_{i-1}}{\partial \widehat{y}}\right)^{2} z_{i}^{2}\right\} .
\end{aligned}
$$

The derivative of $V_{i}$ becomes

$$
\dot{V}_{i}=-\sum_{j=1}^{i-1}\left(c_{j} z_{j}^{2}+d_{j}\left(\frac{\partial \alpha_{j-1}}{\partial \widehat{y}}\right)^{2} z_{j}^{2}+g_{j}\left(\frac{\partial \alpha_{j-1}}{\partial \widehat{y}}\right)^{2} z_{j}^{2}\right)+\tau_{i} \Phi-\sum_{j=1}^{i-1} z_{j} \frac{\partial \alpha_{j-1}}{\partial \widehat{y}}\left(\varepsilon_{\widehat{z}_{12}}+\widehat{d}_{s i_{1}}+\dot{\hat{d}}_{y}\right),
$$

where $\tau_{i} \Phi=\tau_{1} \Phi-\sum_{j=2}^{i-1}\left(\partial \alpha_{j-1} / \partial \widehat{y}\right)\left(z_{j} v_{\ell, 1} \Phi_{\ell}+z_{j} \bar{\omega}^{T} \Phi\right)$.

Step 3. With respect to the new set of coordinates (4.4), the third equation of (4.3) can be written as

$$
\begin{gathered}
\dot{z}_{r}=-k_{r} v_{\ell, 1}+w_{1 \ell}+w_{2 \ell} \widehat{u}-\left[\frac{\partial \alpha_{r-1}}{\partial \widehat{y}}\left(\xi_{12}+v_{\ell, 2} \phi_{\ell}+\bar{\omega}^{T} \Theta+\varepsilon_{\bar{z}_{12}}+\widehat{d}_{s i_{1}}+\dot{\hat{d}}_{y}\right)+\frac{\partial \alpha_{r-1}}{\partial \xi}\left(A_{0} \xi+\bar{k} \hat{y}\right)\right. \\
\left.+\frac{\partial \alpha_{r-1}}{\partial \widetilde{\Theta}} \dot{\Theta} \sum_{j=1}^{\ell} \frac{\partial \alpha_{r-1}}{\partial v_{j}}\left(A_{0} v_{j}+e_{r} \sigma_{j}\right)+\sum_{j=1}^{r-1} \frac{\partial \alpha_{r-1}}{\partial \widehat{y}_{m}^{(j-1)}} \widehat{y}_{m}^{(j)}\right] .
\end{gathered}
$$


The overall Lyapunov function may now be chosen as

$$
V_{r}=\sum_{j=1}^{r-1} V_{j}+\frac{1}{2} z_{r}^{2}+\frac{1}{2} \Phi^{T} \Gamma^{-1} \Phi+\sum_{j=1}^{r} \frac{1}{4 d_{j}} \varepsilon^{T} P \varepsilon
$$

where $\Gamma$ is a symmetric positive definite matrix, that is, $\Gamma=\Gamma^{T}>0$. With the definition of state estimated error $\varepsilon$, we can obtain that

$$
\begin{aligned}
\dot{V}_{r}=\sum_{j=1}^{r-1} \dot{V}_{j}+z_{r}\left\{-k_{r} v_{\ell, 1}+w_{1 \ell}+w_{2 \ell} \widehat{u}-\right. & {\left[\frac{\partial \alpha_{r-1}}{\partial \widehat{y}}\left(\xi_{12}+v_{\ell, 2} \phi_{\ell}+\bar{\omega}^{T} \Theta+\varepsilon_{\bar{z}_{12}}+\widehat{d}_{s i_{1}}+\dot{\hat{d}}_{y}\right)\right.} \\
+ & \frac{\partial \alpha_{r-1}}{\partial \xi}\left(A_{0} \xi+\bar{k} \widehat{y}\right)+\frac{\partial \alpha_{r-1}}{\partial \widetilde{\Theta}} \sum_{j=1}^{\ell} \frac{\partial \alpha_{r-1}}{\partial v_{j}}\left(A_{0} v_{j}+e_{r} \sigma_{j}\right) \\
& \left.\left.+\sum_{j=1}^{r-1} \frac{\partial \alpha_{r-1}}{\partial \widehat{y}_{m}^{(j-1)}} \widehat{y}_{m}^{(j)}\right]\right\} \\
+ & \dot{\Phi}^{T} \Gamma^{-1} \Phi-\sum_{j=1}^{r} \frac{1}{4 d_{j}} \varepsilon^{T} \varepsilon+\sum_{j=1}^{r} \frac{1}{4 d_{j}}\left(\varepsilon^{T} P \Delta+\Delta^{T} P \varepsilon\right) .
\end{aligned}
$$

Specify the control input as

$$
\begin{aligned}
\widehat{u}=\frac{1}{z_{r} w_{2 \ell}}\left\{z_{r} k_{r} v_{\ell, 1}-z_{r} w_{1 \ell}+z_{r}\right. & {\left[\frac{\partial \alpha_{r-1}}{\partial \widehat{y}}\left(\xi_{12}+v_{\ell, 2} \tilde{\phi}_{\ell}+\bar{\omega}^{T} \widetilde{\Theta}\right)+\frac{\partial \alpha_{r-1}}{\partial \xi}\left(A_{0} \xi+\bar{k} \widehat{y}\right)\right.} \\
& \left.+\frac{\partial \alpha_{r-1}}{\partial \widetilde{\Theta}} \dot{\tilde{\Theta}}_{j=1}^{\ell} \frac{\partial \alpha_{r-1}}{\partial v_{j}}\left(A_{0} v_{j}+e_{r} \sigma_{j}\right)+\sum_{j=1}^{r-1} \frac{\partial \alpha_{r-1}}{\partial \widehat{y}_{m}^{(j-1)}} \widehat{y}_{m}^{(j)}\right] \\
& \left.-c_{r} z_{r}^{2}-d_{r}\left(\frac{\partial \alpha_{r-1}}{\partial \widehat{y}}\right)^{2} z_{r}^{2}-g_{r}\left(\frac{\partial \alpha_{r-1}}{\partial \widehat{y}}\right)^{2} z_{r}^{2}+z_{r} \widehat{\hat{u}_{\hat{R}}}\right\}
\end{aligned}
$$

where $\widehat{u}_{\widehat{R}}$ is an addition input which will be used to target on rejection of uncertainties.

Substituting (4.16) into $\dot{V}_{r}$, we have

$$
\begin{aligned}
\dot{V}_{r}= & -\sum_{j=1}^{r}\left(c_{j} z_{j}^{2}+d_{j}\left(\frac{\partial \alpha_{j-1}}{\partial \widehat{y}}\right)^{2} z_{j}^{2}+g_{j}\left(\frac{\partial \alpha_{j-1}}{\partial \widehat{y}}\right)^{2} z_{j}^{2}\right)+\tau_{r-1} \Phi-\frac{\partial \alpha_{r-1}}{\partial \widehat{y}}\left(z_{r} v_{\ell, 1} \Phi_{\ell}+z_{r} \bar{\omega}^{T} \Phi\right)+z_{r} \widehat{u}_{\widehat{R}} \\
& -\sum_{j=1}^{r} z_{j} \frac{\partial \alpha_{j-1}}{\partial \widehat{y}}\left(\varepsilon_{\hat{z}_{12}}+\widehat{d}_{s i_{1}}+\dot{\hat{d}}_{y}\right)+\dot{\Phi}^{T} \Gamma^{-1} \Phi-\sum_{j=1}^{r} \frac{1}{4 d_{j}} \varepsilon^{T} \varepsilon+\sum_{j=1}^{r} \frac{1}{4 d_{j}}\left(\varepsilon^{T} P \Delta+\Delta^{T} P \varepsilon\right) .
\end{aligned}
$$


Write $\tau_{r} \Phi=\tau_{r-1} \Phi-\left(\partial \alpha_{r-1} / \partial \widehat{y}\right)\left(z_{r} v_{\ell, 1} \Phi_{\ell}+z_{r} \bar{\omega}^{T} \Phi\right)$ and we arrive at

$$
\begin{aligned}
\dot{V}_{r}= & -\sum_{j=1}^{r}\left(c_{j} z_{j}^{2}+d_{j}\left(\frac{\partial \alpha_{j-1}}{\partial \widehat{y}}\right)^{2} z_{j}^{2}+g_{j}\left(\frac{\partial \alpha_{j-1}}{\partial \widehat{y}}\right)^{2} z_{j}^{2}\right)+\left(\tau_{r}+\dot{\Phi}^{T} \Gamma^{-1}\right) \Phi+z_{r} \widehat{u}_{\widehat{R}} \\
& -\sum_{j=1}^{r} z_{j} \frac{\partial \alpha_{j-1}}{\partial \widehat{y}}\left(\varepsilon_{\hat{z}_{12}}+\widehat{d}_{s i_{1}}+\dot{\hat{d}}_{y}\right)-\sum_{j=1}^{r} \frac{1}{4 d_{j}} \varepsilon^{T} \varepsilon+\sum_{j=1}^{r} \frac{1}{4 d_{j}}\left(\varepsilon^{T} P \Delta+\Delta^{T} P \varepsilon\right) .
\end{aligned}
$$

From (4.18), we may specify the parameter update law in order to cancel the term $\left(\tau_{r}+\right.$ $\left.\dot{\Phi}^{T} \Gamma^{-1}\right) \Phi$. To guarantee the estimated parameters will always lie within allowable region $w$, a projected parametric update law will be specified as

$$
\dot{\tilde{\Theta}}= \begin{cases}\Gamma \tau_{r}^{T} & \text { if } \widetilde{\Theta} \in w^{0}, \\ P_{R}\left(\Gamma \tau_{r}^{T}\right) & \text { if } \widehat{\Theta} \in \partial w, \tau_{r} \Gamma \widetilde{\Theta}_{\text {perp }}>0,\end{cases}
$$

where $w$ is the allowable parameter variation set (compact and convex) with its interior and boundary denoted by $w^{0}$ and $\partial w$, respectively. If the current estimated parameter vector lies within the allowable parameter variation set, normal update law is employed. If the current estimated parameter vector lies on the boundary of the allowable parameter variation set, projected update law denoted by $P_{R}(\cdot)$ is employed to prevent the parameter vector from leaving the variation set. With (4.19), (4.18) can be written as

$$
\begin{aligned}
\dot{V}_{r}= & -\sum_{j=1}^{r}\left(c_{j} z_{j}^{2}+g_{j}\left(\frac{\partial \alpha_{j-1}}{\partial \widehat{y}}\right)^{2} z_{j}^{2}\right)-\sum_{j=1}^{r} d_{j}\left(\frac{\partial \alpha_{j-1}}{\partial \widehat{y}} z_{i}+\frac{1}{2 d_{j}} \varepsilon_{\widehat{z}_{12}}\right)^{2}+z_{r} \widehat{u}_{\widehat{R}} \\
& -\sum_{j=1}^{r} z_{j} \frac{\partial \alpha_{j-1}}{\partial \widehat{y}}\left(\widehat{d}_{s i_{1}}+\hat{d}_{y}\right)-\sum_{j=1}^{r} \frac{1}{4 d_{j}}\left(\varepsilon_{\hat{z}_{11}}^{2}+\varepsilon_{\hat{z}_{13}}^{2}+\cdots+\varepsilon_{\hat{z}_{1 r}}^{2}\right)+\sum_{j=1}^{r} \frac{1}{4 d_{j}}\left(\varepsilon^{T} P \Delta+\Delta^{T} P \varepsilon\right) \\
\leq & -\sum_{j=1}^{r}\left(c_{j} z_{j}^{2}+g_{j}\left(\frac{\partial \alpha_{j-1}}{\partial \widehat{y}}\right)^{2} z_{j}^{2}\right)-\sum_{j=1}^{r} d_{j}\left(\frac{\partial \alpha_{j-1}}{\partial \widehat{y}} z_{j}+\frac{1}{2 d_{j}} \varepsilon_{\hat{z}_{12}}\right)^{2}+z_{r} \widehat{u}_{\widehat{R}} \\
& -\sum_{j=1}^{r} z_{j} \frac{\partial \alpha_{j-1}}{\partial \widehat{y}}\left|\widehat{d}_{s i_{1}}+\hat{\widehat{d}}_{y}\right|-\sum_{j=1}^{r} \frac{1}{4 d_{j}}\left(\varepsilon_{\hat{z}_{11}}^{2}+\varepsilon_{\hat{z}_{13}}^{2}+\cdots+\varepsilon_{\hat{z}_{1 r}}^{2}\right)+\sum_{j=1}^{r} \frac{1}{4 d_{j}}\left(\varepsilon^{T} P \Delta+\Delta^{T} P \varepsilon\right) .
\end{aligned}
$$


Add and subtract terms $\sum_{j=1}^{r}\left(1 / 4 g_{j}\right)\left|\widehat{d}_{s i_{1}}+\dot{\hat{d}}_{y}\right|^{2}$; we have

$$
\begin{aligned}
\dot{V}_{r} \leq & -\sum_{j=1}^{r} c_{j} z_{j}^{2}-\sum_{j=1}^{r} d_{j}\left(\frac{\partial \alpha_{j-1}}{\partial \widehat{y}} z_{j}+\frac{1}{2 d_{j}} \varepsilon_{\hat{z}_{12}}\right)^{2}+z_{r} \widehat{u}_{\widehat{R}} \\
& -\sum_{j=1}^{r} g_{j}\left(\frac{\partial \alpha_{j-1}}{\partial \hat{y}}\right)^{2} z_{j}^{2}-\sum_{j=1}^{r} z_{j} \frac{\partial \alpha_{j-1}}{\partial \widehat{y}}\left|\widehat{d}_{s i_{1}}+\dot{\hat{d}}_{y}\right|-\sum_{j=1}^{r} \frac{1}{4 g_{j}}\left|\widehat{d}_{s i_{1}}+\dot{\hat{d}}_{y}\right|^{2} \\
& +\sum_{j=1}^{r} \frac{1}{4 g_{j}}\left|\widehat{d}_{s i_{1}}+\dot{\hat{d}}_{y}\right|^{2}+\sum_{j=1}^{r} \frac{1}{4 d_{j}}\left(\varepsilon^{T} P \Delta+\Delta^{T} P \varepsilon\right)-\sum_{j=1}^{r} \frac{1}{4 d_{j}}\left(\varepsilon_{\hat{z}_{11}}^{2}+\varepsilon_{\hat{z}_{13}}^{2}+\cdots+\varepsilon_{\hat{z}_{1 r}}^{2}\right) .
\end{aligned}
$$

Moreover, we obtain

$$
\begin{aligned}
\dot{V}_{i} \leq & -\sum_{j=1}^{r} c_{j} z_{j}^{2}-\sum_{j=1}^{r} d_{j}\left(\frac{\partial \alpha_{j-1}}{\partial \widehat{y}} z_{j}+\frac{1}{2 d_{j}} \varepsilon_{\hat{z}_{12}}\right)^{2}-\sum_{j=1}^{r} g_{j}\left(\frac{\partial \alpha_{j-1}}{\partial \widehat{y}} z_{j}+\frac{1}{2 g_{j}}\left|\widehat{d}_{s i_{1}}+\hat{\widehat{d}}_{y}\right|\right)^{2} \\
& +\sum_{j=1}^{r} \frac{1}{4 g_{j}}\left|\widehat{d}_{s i_{1}}+\dot{\hat{d}}_{y}\right|^{2}+\sum_{j=1}^{r} \frac{1}{4 d_{i}}\left(\varepsilon^{T} P \Delta+\Delta^{T} P \varepsilon\right)-\sum_{j=1}^{r} \frac{1}{4 d_{j}}\left(\varepsilon_{\hat{z}_{11}}^{2}+\varepsilon_{\hat{z}_{13}}^{2}+\cdots+\varepsilon_{\hat{z}_{1 r}}^{2}\right)+z_{r} \widehat{u}_{\hat{R}} .
\end{aligned}
$$

As shown in Figure 1, the tracking error $Z_{1}(\widetilde{S})$ and the control input $\widehat{U}_{\widehat{R}}(\widetilde{s})$ are related by

$$
\widehat{U}_{\widehat{R}}(\widetilde{S})=-\widehat{R}(\widetilde{S}) \widehat{C}(\widetilde{S}) Z_{1}(\widetilde{S}),
$$

where we have chosen $\widehat{R}(\widetilde{s})$ as a low-order and attenuated-type internal model filter, that is,

$$
\widehat{R}(\widetilde{s})=\prod_{i=1}^{k} \frac{\widetilde{s}^{2}+2 \zeta_{i} \omega_{n i} \widetilde{s}+\omega_{n i}^{2}}{\widetilde{s}^{2}+2 \xi_{i} \omega_{n i} \widetilde{s}+\omega_{n i}^{2}}
$$

where $k$ is the number of periodic frequencies to be rejected, $\omega_{n i}$ is determined based on the $i$ th disturbance frequency in $\mathrm{rad} / \mathrm{rev}$, and $\xi_{i}$ and $\zeta_{i}$ are two damping ratios that satisfy $0<\xi_{i}<\zeta_{i}<1$. We can adjust the gain of $\widehat{R}(\widetilde{s})$ at those periodic frequencies by varying the values of $\xi_{i}$ and $\zeta_{i}$.

Theorem 4.1. Consider the control law of (4.16) and (4.23) applied to a nonlinear system with unmodeled dynamics, parameter uncertainty and subject to output disturbance as given by (3.1). Assume that $\widehat{y}_{m}, \dot{\hat{y}}_{m}, \ldots, \widehat{y}_{m}^{(r)}$ (where $r$ is the relative degree) and $\widehat{d}_{y}, \hat{\vec{d}}_{y}, \ldots, \widehat{d}_{y}^{(r)}$ are known and bounded, $\widehat{d}_{s i_{1}}^{(r-1)}, \widehat{d}_{s i_{2}}^{(r-2)}, \ldots, \hat{\vec{d}}_{s i_{r-1}}$ are sufficiently smooth, $f, g, h, L_{f}^{r} h, L_{g} L_{f}^{r-1} h$ are Lipschitz continuous functions, at least one column of $\bar{W}(\widehat{y})$ is bounded away from zero. Furthermore, suppose that a loop-shaping filter $\widehat{C}(\widetilde{s})$ is designed such that the feedback system is stable. Then the modified parameter update law as given by (4.19) yields the bounded tracking error. 


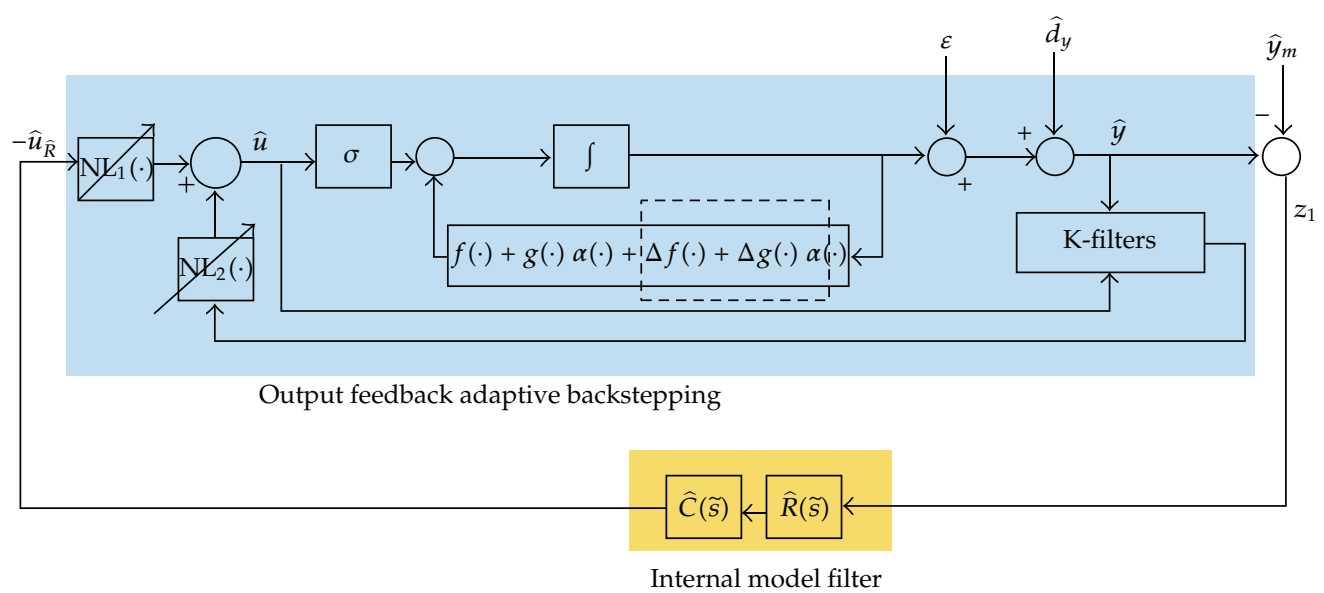

Figure 1: The control configuration for the proposed spatial domain adaptive control system.

Proof. Step 1 (show that only $\widetilde{\Theta} \in \mathrm{w}^{0}$ needs to be considered).

Denote by $\dot{\widetilde{\Theta}}_{\text {perp }}$ the component of $\dot{\tilde{\Theta}}$ perpendicular to the tangent plane at $\widetilde{\Theta}$ so that $\dot{\tilde{\Theta}}=P_{R}(\dot{\tilde{\Theta}})+\dot{\tilde{\Theta}}_{\text {perp }}$. Since $\Theta \in w$ and $w$ is convex, we have $(\widetilde{\Theta}-\Theta)^{T} \dot{\tilde{\Theta}}_{\text {perp }} \geq 0$. Choose Lyapunov function $V(\Phi)=\Phi^{T} \Phi$ and use the parameter update law as defined in (4.19). When $\widetilde{\Theta} \in w^{0}$, we have $\dot{V}=-\Phi^{T} \dot{\tilde{\Theta}}$. When $\widetilde{\Theta} \in \partial w$, we have

$$
\dot{V}=-\Phi^{T} P_{R}(\dot{\tilde{\Theta}})=-\Phi^{T}\left(\dot{\tilde{\Theta}}-\dot{\tilde{\Theta}}_{\text {perp }}\right)=-\Phi^{T} \dot{\tilde{\Theta}}+\Phi^{T} \dot{\tilde{\Theta}}_{\text {perp }} \leq-\Phi^{T} \dot{\tilde{\Theta}}
$$

where we use the fact that

$$
\Phi^{T} \dot{\widetilde{\Theta}}_{\text {perp }}=(\Theta-\widetilde{\Theta})^{T} \dot{\widetilde{\Theta}}_{\text {perp }}=-(\widetilde{\Theta}-\Theta)^{T} \dot{\tilde{\Theta}}_{\text {perp }} \leq 0 .
$$

Thus, we only have to consider the scenario corresponding to $\widetilde{\Theta} \in w^{0}$ in the sequel.

Step 2. Substituting (4.23) back into (4.22), we have

$$
\begin{aligned}
\dot{V}_{r} \leq & -\sum_{j=1}^{r} c_{j} z_{j}^{2}-\sum_{j=1}^{r} d_{j}\left(\frac{\partial \alpha_{j-1}}{\partial \widehat{y}} z_{j}+\frac{1}{2 d_{j}} \varepsilon_{\widehat{z}_{12}}\right)^{2}-\sum_{j=1}^{r} g_{j}\left(\frac{\partial \alpha_{j-1}}{\partial \widehat{y}} z_{j}+\frac{1}{2 g_{j}}\left|\widehat{d}_{s i_{1}}+\dot{\hat{d}}_{y}\right|\right)^{2} \\
& +\sum_{j=1}^{r} \frac{1}{4 g_{j}}\left|\widehat{d}_{s i_{1}}+\dot{\hat{d}}_{y}\right|^{2}+\sum_{j=1}^{r} \frac{1}{4 d_{j}}\left(\varepsilon^{T} P \Delta+\Delta^{T} P \varepsilon\right) \\
& -\sum_{j=1}^{r} \frac{1}{4 d_{j}}\left(\varepsilon_{\hat{z}_{11}}^{2}+\varepsilon_{\hat{z}_{13}}^{2}+\cdots+\varepsilon_{\hat{z}_{1 r}}^{2}\right)-z_{r} \widehat{R}(\widetilde{s}) \widehat{C}(\widetilde{s}) z_{1} .
\end{aligned}
$$

Using the definition of tracking error $z_{1}=\widehat{y}-\widehat{y}_{m}=\left(\bar{z}_{11}-\widehat{y}_{m}\right)+\varepsilon_{\hat{z}_{11}}-\widehat{d}_{y}$, (4.27) can be 
written as

$$
\begin{aligned}
\dot{V}_{r} \leq & -\sum_{j=1}^{r} c_{j} z_{j}^{2}-\sum_{j=1}^{r} d_{j}\left(\frac{\partial \alpha_{j-1}}{\partial \widehat{y}} z_{j}+\frac{1}{2 d_{j}} \varepsilon_{\widehat{z}_{12}}\right)^{2}-\sum_{j=1}^{r} g_{j}\left(\frac{\partial \alpha_{j-1}}{\partial \widehat{y}} z_{j}+\frac{1}{2 g_{j}}\left|\widehat{d}_{s i_{1}}+\dot{\widehat{d}}_{y}\right|\right)^{2} \\
& -\sum_{j=1}^{r} \frac{1}{4 d_{j}}\left(\varepsilon_{\hat{z}_{11}}^{2}+\varepsilon_{\hat{z}_{13}}^{2}+\cdots+\varepsilon_{\hat{z}_{1 r}}^{2}\right)+\sum_{j=1}^{r} \frac{1}{4 g_{j}}\left|\widehat{d}_{s i_{1}}+\dot{\hat{d}}_{y}\right|^{2}+\sum_{j=1}^{r} \frac{1}{4 d_{j}}\left(\varepsilon^{T} P \Delta+\Delta^{T} P \varepsilon\right) \\
& +\left|z_{r} \widehat{R}(\widetilde{s}) \widehat{C}(\widetilde{s})\left(\bar{z}_{11}-\widehat{y}_{m}\right)\right|+\left|z_{r} \widehat{R}(\widetilde{s}) \widehat{C}(\widetilde{s})\left(\varepsilon_{\widehat{z}_{11}}-\widehat{d}_{y}\right)\right| .
\end{aligned}
$$

Use the following equality:

$$
\widehat{z}_{r} \widehat{R}(\widetilde{s}) \widehat{C}(\widetilde{s})\left(\varepsilon_{\hat{z}_{11}}-\widehat{d}_{y}\right) \leq \gamma^{2} \widehat{z}_{r}^{2}+\left(\frac{1}{2 \gamma} \widehat{R}(\widetilde{s}) \widehat{C}(\widetilde{s})\left(\varepsilon_{\widehat{z}_{11}}-\widehat{d}_{y}\right)\right)^{2}, \quad \gamma>0 \text { is designable. }
$$

Equation (4.28) becomes

$$
\begin{aligned}
\dot{V}_{r} \leq & -\sum_{j=1}^{r-1} c_{j} z_{j}^{2}-c_{r}^{\prime} z_{r}^{2}-\sum_{j=1}^{r} d_{j}\left(\frac{\partial \alpha_{j-1}}{\partial \widehat{y}} z_{j}+\frac{1}{2 d_{j}} \varepsilon_{\hat{z}_{12}}\right)^{2}-\sum_{j=1}^{r} g_{j}\left(\frac{\partial \alpha_{j-1}}{\partial \widehat{y}} z_{j}+\frac{1}{2 g_{j}}\left|\widehat{d}_{s i_{1}}+\dot{\hat{d}}_{y}\right|\right)^{2} \\
& +\sum_{j=1}^{r} \frac{1}{4 g_{j}}\left|\widehat{d}_{s i_{1}}+\hat{\widehat{d}}_{y}\right|^{2}+\sum_{j=1}^{r} \frac{1}{4 d_{j}}\left(\varepsilon^{T} P \Delta+\Delta^{T} P \varepsilon\right)+\left|z_{r} \widehat{R}(\widetilde{s}) \widehat{C}(\widetilde{s})\left(\bar{z}_{11}-\widehat{y}_{m}\right)\right| \\
& +\left(\frac{1}{2 \gamma} \widehat{R}(\widetilde{s}) \widehat{C}(\widetilde{s})\left(\varepsilon_{\hat{z}_{11}}-\widehat{d}_{y}\right)\right)^{2}-\sum_{j=1}^{r} \frac{1}{4 d_{j}}\left(\varepsilon_{\hat{z}_{11}}^{2}+\varepsilon_{\hat{z}_{13}}^{2}+\cdots+\varepsilon_{\hat{z}_{1 r}}^{2}\right),
\end{aligned}
$$

where $c_{r}^{\prime}=c_{r}-\gamma^{2}>0$. Moreover, the positive designable parameters $c_{i}$ can be written as

$$
\begin{gathered}
c_{j}=C_{j}+h_{j}, \quad j=1, \ldots, r-1, \\
c_{r}^{\prime}=C_{r}+h_{r},
\end{gathered}
$$

where $C_{j}, C_{r}$ and $h_{j}, h_{r}>0$. Thus, (4.30) can be written as

$$
\begin{aligned}
\dot{V}_{r} \leq & -\sum_{j=1}^{r-1}\left(C_{j}+h_{j}\right) z_{j}^{2}-\left(C_{r}+h_{r}\right) z_{r}^{2}-\sum_{j=1}^{r} d_{j}\left(\frac{\partial \alpha_{j-1}}{\partial \widehat{y}} z_{j}+\frac{1}{2 d_{j}} \varepsilon_{\hat{z}_{12}}\right)^{2} \\
& -\sum_{j=1}^{r} g_{j}\left(\frac{\partial \alpha_{j-1}}{\partial \widehat{y}} z_{j}+\frac{1}{2 g_{j}}\left|\widehat{d}_{s i_{1}}+\dot{\hat{d}}_{y}\right|\right)^{2}-\sum_{j=1}^{r} \frac{1}{4 d_{j}}\left(\varepsilon_{\hat{z}_{11}}^{2}+\varepsilon_{\bar{z}_{13}}^{2}+\cdots+\varepsilon_{\bar{z}_{1 r}}^{2}\right) \\
& +\sum_{j=1}^{r} \frac{1}{4 g_{j}}\left|\widehat{d}_{s i_{1}}+\dot{\hat{d}}_{y}\right|^{2}+\sum_{j=1}^{r} \frac{1}{4 d_{j}}\left(\varepsilon^{T} P \Delta+\Delta^{T} P \varepsilon\right)+\left|z_{r} \widehat{R}(\widetilde{s}) \widehat{C}(\widetilde{s})\left(\bar{z}_{11}-\widehat{y}_{m}\right)\right| \\
& +\left(\frac{1}{2 \gamma} \widehat{R}(\widetilde{S}) \widehat{C}(\widetilde{s})\left(\varepsilon_{\hat{z}_{11}}-\widehat{d}_{y}\right)\right)^{2} .
\end{aligned}
$$


Journal of Applied Mathematics

Utilizing the fact that

$$
\left(\sqrt{h_{1}}\left|z_{1}\right|-\sqrt{h_{r}}\left|z_{r}\right|\right)^{2}=z_{1}^{2}+h_{r} z_{r}^{2}-\sqrt{h_{1} h_{r}}\left|z_{r}\left(\bar{z}_{11}-\widehat{y}_{m}\right)+z_{r}\left(\varepsilon_{\widehat{z}_{11}}-\widehat{d}_{y}\right)\right|,
$$

we have

$$
\begin{aligned}
\dot{V}_{r} \leq & -\sum_{j=2}^{r-1}\left(C_{j}+h_{j}\right) z_{j}^{2}-C_{1} z_{1}^{2}-C_{r} z_{r}^{2} \\
& -\left(\sqrt{h_{1}}\left|z_{1}\right|-\sqrt{h_{r}}\left|z_{r}\right|\right)^{2}-\sqrt{h_{1} h_{r}}\left|z_{r}\left(\bar{z}_{11}-\widehat{y}_{m}\right)+z_{r}\left(\varepsilon_{\hat{z}_{11}}-\widehat{d}_{y}\right)\right| \\
& -\sum_{j=1}^{r} d_{j}\left(\frac{\partial \alpha_{j-1}}{\partial \widehat{y}} z_{j}+\frac{1}{2 d_{j}} \varepsilon_{\hat{z}_{12}}\right)^{2}-\sum_{j=1}^{r} g_{j}\left(\frac{\partial \alpha_{j-1}}{\partial \widehat{y}} z_{j}+\frac{1}{2 g_{j}}\left|\widehat{d}_{s i_{1}}+\dot{\hat{d}}_{y}\right|\right)^{2} \\
& -\sum_{j=1}^{r} \frac{1}{4 d_{j}}\left(\varepsilon_{\hat{z}_{11}}^{2}+\varepsilon_{\hat{z}_{13}}^{2}+\cdots+\varepsilon_{\hat{z}_{1 r}}^{2}\right)+\sum_{j=1}^{r} \frac{1}{4 g_{j}}\left|\widehat{d}_{s i_{1}}+\dot{\hat{d}}_{y}\right|^{2}+\sum_{j=1}^{r} \frac{1}{4 d_{j}}\left(\varepsilon^{T} P \Delta+\Delta^{T} P \varepsilon\right) \\
& +\left|z_{r} \widehat{R}(\widetilde{s}) \widehat{C}(\widetilde{s})\left(\bar{z}_{11}-\widehat{y}_{m}\right)\right|+\left(\frac{1}{2 \gamma} \widehat{R}(\widetilde{s}) \widehat{C}(\widetilde{s})\left(\varepsilon_{\widehat{z}_{11}}-\widehat{d}_{y}\right)\right)^{2} .
\end{aligned}
$$

To design $h_{1}, \ldots, h_{r}$ (or $\left.c_{1}, \ldots, c_{r}\right), d_{j}$ and $g_{j}$ such that

$$
\begin{aligned}
& -\sum_{j=1}^{r} h_{j} z_{j}^{2}-\sum_{j=1}^{r} d_{j}\left(\frac{\partial \alpha_{j-1}}{\partial \widehat{y}} z_{j}+\frac{1}{2 d_{j}} \varepsilon_{\hat{z}_{12}}\right)^{2}-\sum_{j=1}^{r} g_{j}\left(\frac{\partial \alpha_{j-1}}{\partial \widehat{y}} z_{j}+\frac{1}{2 g_{j}}\left|\widehat{d}_{s i_{1}}+\hat{\bar{d}}_{y}\right|\right)^{2} \\
& -\sum_{j=1}^{r} \frac{1}{4 d_{j}}\left(\varepsilon_{\hat{z}_{11}}^{2}+\varepsilon_{\hat{z}_{13}}^{2}+\cdots+\varepsilon_{\hat{z}_{1 r}}^{2}\right)-\left(\sqrt{h_{1}}\left|z_{1}\right|-\sqrt{h_{r}}\left|z_{r}\right|\right)^{2} \\
& -\sqrt{h_{1} h_{r}}\left|z_{r}\left(\bar{z}_{11}-\widehat{y}_{m}\right)+z_{r}\left(\varepsilon_{\hat{z}_{11}}-\widehat{d}_{y}\right)\right|+\left|z_{r} \widehat{R}(\widetilde{s}) \widehat{C}(\widetilde{s})\left(\bar{z}_{11}-\widehat{y}_{m}\right)\right| \leq 0
\end{aligned}
$$

we arrive at

$$
\dot{V}_{r} \leq-\sum_{j=1}^{r} C_{j} z_{j}^{2}+\left(\frac{1}{2 \gamma} \widehat{R}(\widetilde{S}) \widehat{C}(\widetilde{S})\left(\varepsilon_{\bar{z}_{11}}-\widehat{d}_{y}\right)\right)^{2}+\sum_{j=1}^{r} \frac{1}{4 g_{j}}\left|\widehat{d}_{s i_{1}}+\dot{\hat{d}}_{y}\right|^{2}+\sum_{j=1}^{r} \frac{1}{4 d_{j}}\left(\varepsilon^{T} P \Delta+\Delta^{T} P \varepsilon\right) .
$$

Equation (4.36) implies that

$$
\begin{aligned}
\dot{V}_{r} \leq & -\sum_{j=1}^{r} C_{j} z_{j}^{2}-\left(\frac{1}{2} \Phi^{T} \Gamma^{-1} \Phi+\sum_{j=1}^{r} \frac{1}{4 d_{j}} \varepsilon^{T} P \varepsilon\right)+\left(\frac{1}{2} \Phi^{T} \Gamma^{-1} \Phi+\sum_{j=1}^{r} \frac{1}{4 d_{j}} \varepsilon^{T} P \varepsilon\right) \\
& +\left(\frac{1}{2 \gamma} \widehat{R}(\widetilde{s}) \widehat{C}(\widetilde{s})\left(\varepsilon_{\hat{z}_{11}}-\widehat{d}_{y}\right)\right)^{2}+\sum_{j=1}^{r} \frac{1}{4 g_{j}}\left|\widehat{d}_{s i_{1}}+\hat{\hat{d}}_{y}\right|^{2} \\
& +\sum_{j=1}^{r} \frac{1}{4 d_{j}}\left|\varepsilon^{T} P \Delta+\Delta^{T} P \varepsilon\right| \leq-2 k_{v} V_{r}+C,
\end{aligned}
$$


where $k_{v} \triangleq \min \left\{C_{1}, \ldots C_{r}, \lambda_{\min }(\Gamma)\right\}, \lambda_{\min }(\Gamma)$ is the smallest eigenvalue of $\Gamma$ and

$$
\begin{aligned}
C= & \frac{1}{2} \Phi^{T} \Gamma^{-1} \Phi+\sum_{j=1}^{r} \frac{1}{4 d_{j}} \varepsilon^{T} P \varepsilon+\left(\frac{1}{2 \gamma} \widehat{R}(\widetilde{s}) \widehat{C}(\widetilde{s})\left(\varepsilon_{\widehat{z}_{11}}-\widehat{d}_{y}\right)\right)^{2}+\sum_{j=1}^{r} \frac{1}{4 g_{j}}\left|\widehat{d}_{s i_{1}}+\dot{\hat{d}}_{y}\right|^{2} \\
& +\sum_{j=1}^{r} \frac{1}{4 d_{j}}\left|\varepsilon^{T} P \Delta+\Delta^{T} P \varepsilon\right|
\end{aligned}
$$

is bounded since $\varepsilon_{\widehat{z}_{11}}, \widehat{d}_{y}$ are bounded and $\Phi^{T} \Gamma^{-1} \Phi$ is bounded due to the parameter update law specified in (4.19). We conclude that

$$
V_{r} \leq e^{-2 k_{v} \theta} V_{r}(0)+\int_{0}^{\theta} C e^{-2 k_{v}(\theta-\tau)} d \tau \leq e^{-2 k_{v} \theta} V_{r}(0)+\left(1-e^{-2 k_{v} \theta}\right) \frac{C}{2 k_{v}} .
$$

As $\theta \rightarrow \infty$, we have

$$
V_{r}(\infty) \leq \frac{C}{2 k_{v}}
$$

which implies that the overall system is stable and the bound $C /\left(2 k_{v}\right)$ can be decreased by increasing $k_{v}$ or increasing $\gamma$. By (4.14), this implies that $z, \widetilde{\Theta}, \varepsilon$ are bounded. Since $z_{1}=\widehat{y}-\widehat{y}_{m}, \widehat{y}$ is also bounded. From (3.11), we can see that $\xi$ and $v_{1}, \ldots, v_{\ell}$ are bounded since $\bar{W}_{f}(\widehat{y})$ and $\bar{W}_{g}(\widehat{y})$ are bounded. Moreover, we conclude that the virtual inputs $\alpha$ are bounded because they consist of bounded terms. Also, $\bar{z}_{1}$ is bounded from (3.12) and also $\widehat{z}_{1}$ from the definition of $\varepsilon$. With the ISS assumption and bounded $\widehat{z}_{1}$, we conclude that the internal dynamics $\widehat{z}_{2}$ is bounded. Finally, $\widehat{x}$ is bounded by diffeomorphism, that is, $\widehat{x}=T^{-1}(\widehat{z})$.

\section{Illustrative Example}

For realistic simulation, we set up a simulation configuration as shown in Figure 2, in which the controller and parametric adaptation operate in the $\theta$-domain whereas the open-loop system operates in the time domain. The proposed spatial domain output feedback adaptive control scheme is applied to a reformulated system in spatial domain expressed as

$$
\dot{\hat{x}}=f(\widehat{x})+g(\widehat{x}) \widehat{u}+\widehat{d}_{s}, \quad \widehat{y}=h(\widehat{x})+\widehat{d}_{y}
$$

where

$$
f(\widehat{x})=\left[\begin{array}{c}
-a_{1}+\frac{\widehat{x}_{2}}{\widehat{x}_{1}} \\
-a_{0}
\end{array}\right], \quad g(\widehat{x})=\left[\begin{array}{c}
0 \\
b_{0} \\
\widehat{x}_{1}
\end{array}\right], \quad h(\widehat{x})=\widehat{x}_{1}
$$

with $a_{0}=5155, a_{1}=1138$, and $b_{0}=140368$. For verification purpose, the output disturbance is assumed to be a low-pass rectangular periodic signal (with amplitude switching between 


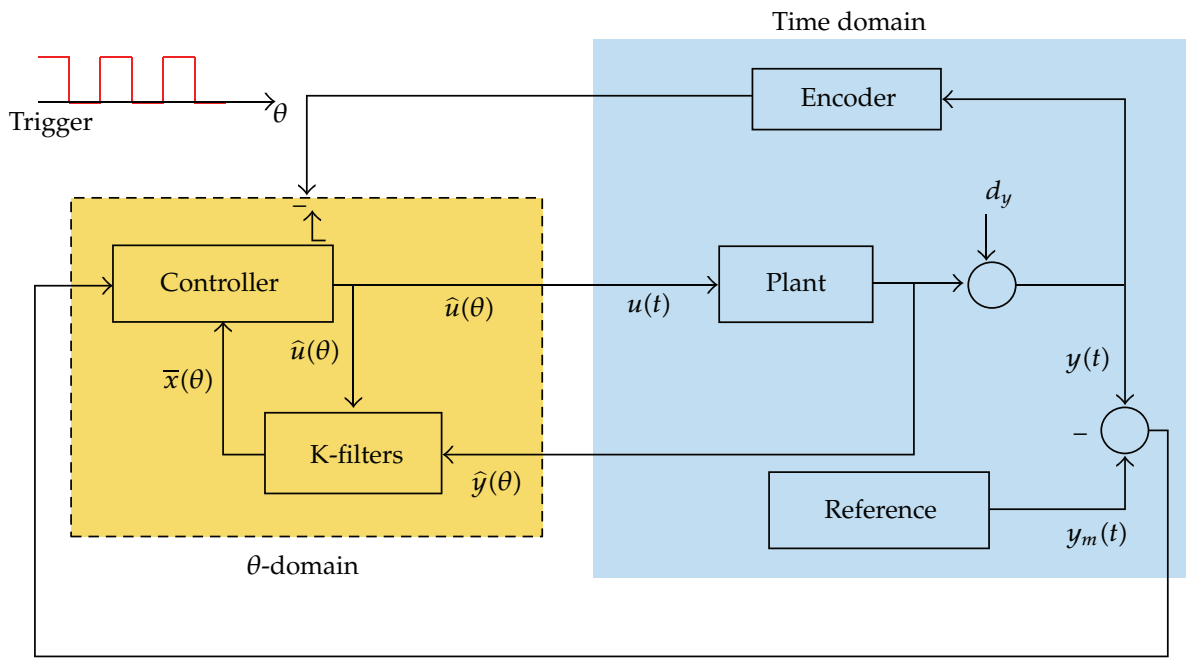

Figure 2: The configuration for numerical simulation.

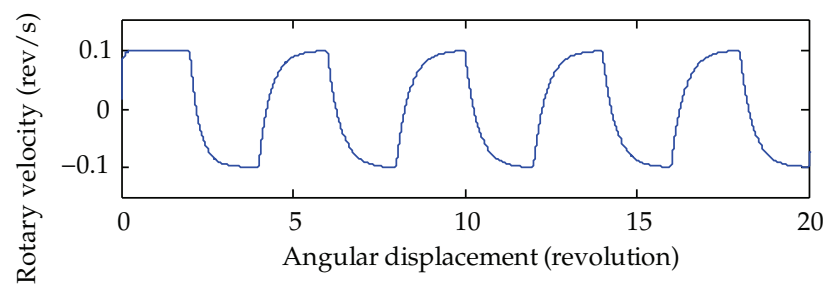

(a)

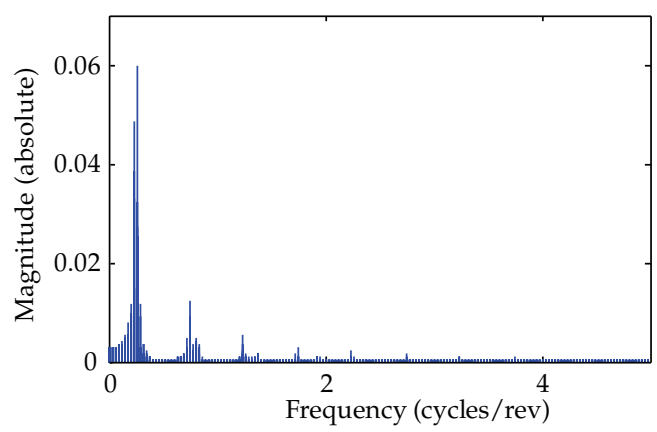

(b)

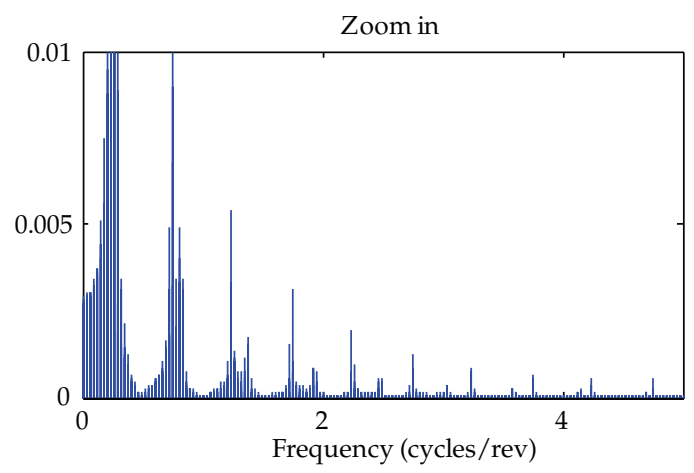

(c)

Figure 3: The output disturbance and the corresponding frequency spectrum.

-0.1 and 0.1 ) (see Figure 3), that is,

$$
\widehat{d}_{y}(\theta)=\frac{0.1}{0.0125 \widetilde{s}+1}\left[\sum_{l=-\infty}^{\infty}(-1)^{l} \Pi(\theta-1-l)\right]+\frac{10}{(0.005 \widetilde{s}+1)^{2}} N_{0}
$$


where

$$
\Pi(\theta)= \begin{cases}1 & |\theta|<1 \\ 0.5 & |\theta|=1 \\ 0 & \text { otherwise }\end{cases}
$$

Note that the disturbance has been low-pass filtered so that it is continuously differentiable. Parameters of the internal model filter are specified to target the fundamental frequency and the first three harmonic frequencies of the periodic disturbance, that is,

$$
\widehat{R}(\widetilde{s})=\prod_{i=1}^{4} \frac{\widetilde{s}^{2}+2 \zeta_{i} \omega_{n i} \widetilde{s}+\omega_{n i}^{2}}{\widetilde{s}^{2}+2 \xi_{i} \omega_{n i} \widetilde{s}+\omega_{n i}^{2}}
$$

where

$$
\begin{array}{cl}
S_{i}=0.2, \quad \xi_{i}=0.0002, & \\
\omega_{n 1}=0.25 \pi, \quad \omega_{n 2}=3 \times 0.25 \pi, & \omega_{n 3}=5 \times 0.25 \pi, \quad \omega_{n 4}=7 \times 0.25 \pi,
\end{array}
$$

Furthermore, the stabilizing filter is specified as

$$
\widehat{C}(\widetilde{s})=\frac{100000(\widetilde{s} / 100+1)}{(\widetilde{s} / 10000+1)} .
$$

The parameters of the $K$-filter are set to $k_{1}=1600$ and $k_{2}=100$. The initial values of the estimated parameters are set to $\tilde{a}_{0}=1500, \tilde{a}_{1}=500$, and $\tilde{b}_{0}=1000000$. The allowable parameter variation sets are

$$
\begin{aligned}
& \tilde{a}_{0} \in \Omega_{\tilde{a}_{0}} \triangleq\left\{\tilde{a}_{0}: 100 \leq \tilde{a}_{0} \leq 10000\right\} \\
& \tilde{a}_{1} \in \Omega_{\tilde{a}_{1}} \triangleq\left\{\tilde{a}_{1}: 10 \leq \tilde{a}_{1} \leq 10000\right\} \\
& \tilde{b}_{0} \in \Omega_{\tilde{b}_{0}} \triangleq\left\{\tilde{b}_{0}: 10000 \leq \tilde{b}_{0} \leq 10000000\right\}
\end{aligned}
$$

Note that $d_{s}(t)$ is set to 0 so that the system performance is not affected by the unstructured uncertainty. Suppose that a variable speed control task demands the system to initially run at $30 \mathrm{rev} / \mathrm{s}$ and then speed up to $35 \mathrm{rev} / \mathrm{s}$ and finally speed down to $25 \mathrm{rev} / \mathrm{s}$ (see Figure 4). To avoid getting infinite value when taking derivative, the reference command is specified to have smooth (instead of instant) change. Figure 5 compares the tracking performance of two scenarios. The figures on the left are for the pure output feedback adaptive backstepping design. The ones on the right are for the proposed output feedback design with internal model control. Without internal model control, the adaptive backstepping design has already shown superb tracking performance. We see that adding the internal model control further reduces the magnitude of the tracking error without noticeable increase in the control input. 


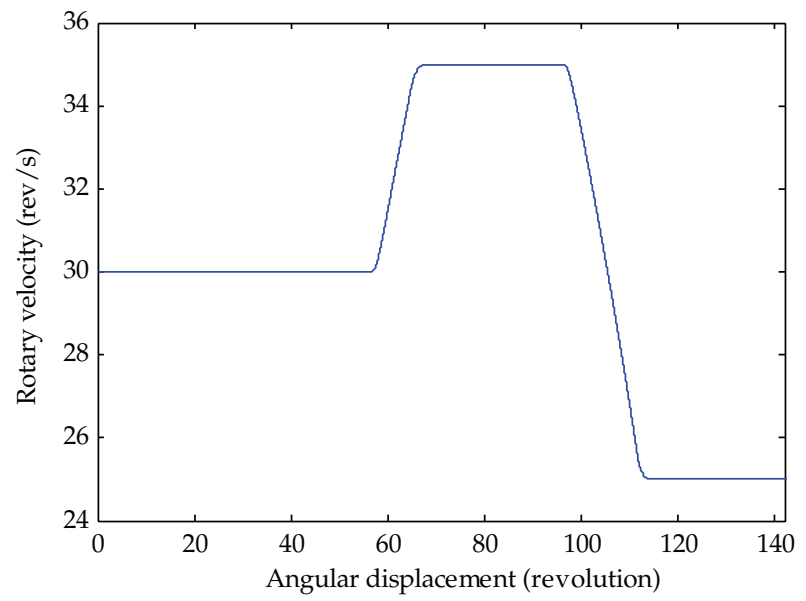

Figure 4: The tracking command for a variable speed control task.

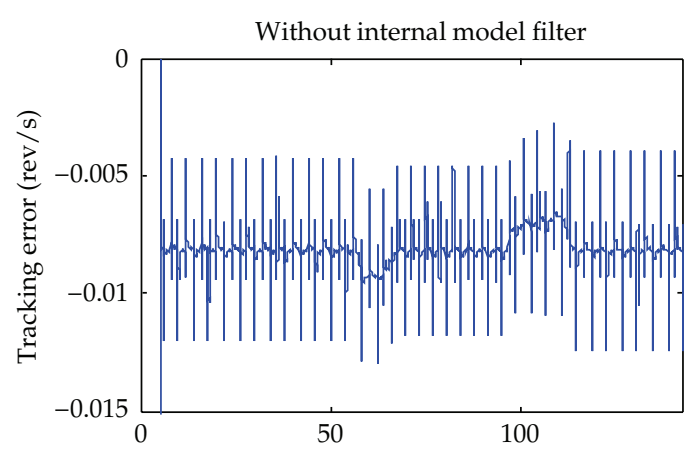

(a)

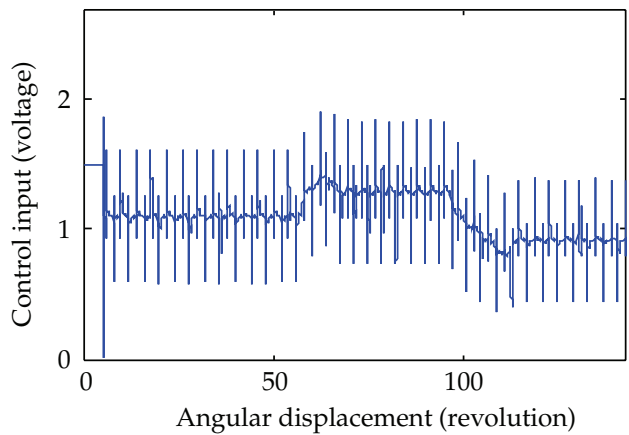

(c)

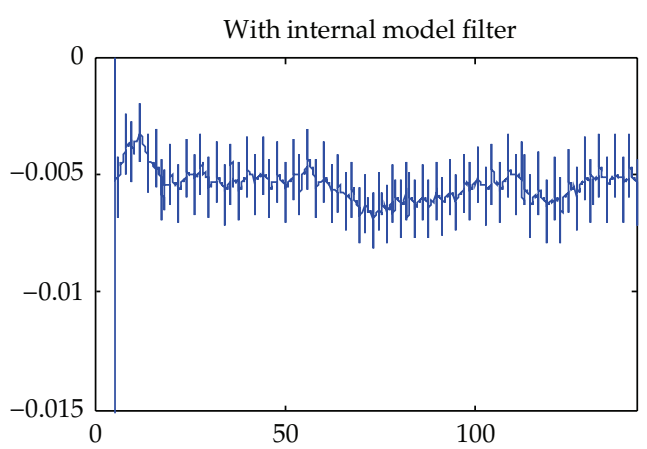

(b)

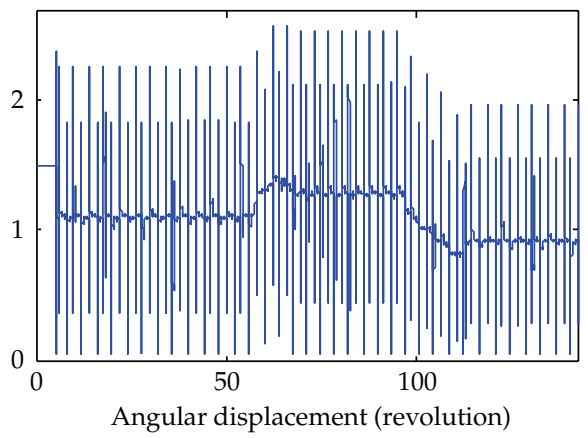

(d)

Figure 5: Comparison of tracking performance.

\section{Conclusion}

This paper presents the design of a new spatial domain adaptive control system, which can be applied to rotary systems operating at varying speeds and subject to spatially periodic and band-limited disturbances and structured/unstructured parametric uncertainties. 
The proposed design integrates two control paradigms, that is, adaptive backstepping and internal model control. The overall output feedback adaptive control system can be shown to be stable and have bounded state estimated error and output tracking error. Feasibility and effectiveness of the proposed design are further justified by a numerical example. Future effort will be dedicated to implementation and verification of the proposed control design to a practical rotary system, for example, a brushless dc-motor-driven control system.

\section{References}

[1] B. A. Francis and W. M. Wonham, "The internal model principle of control theory," Automatica, vol. 12 , no. 5, pp. 457-465, 1976.

[2] V. O. Nikiforov, "Adaptive non-linear tracking with complete compensation of unknown disturbances," European Journal of Control, vol. 4, pp. 132-139, 1998.

[3] Z. Ding, "Adaptive disturbance rejection of nonlinear systems in an extended output feedback form," IET Control Theory \& Applications, vol. 1, no. 1, pp. 298-303, 2007.

[4] L. Marconi, A. Isidori, and A. Serrani, "Input disturbance suppression for a class of feedforward uncertain nonlinear systems," Systems \& Control Letters, vol. 45, no. 3, pp. 227-236, 2002.

[5] L. Gentili and L. Marconi, "Robust nonlinear disturbance suppression of a magnetic levitation system," Automatica, vol. 39, no. 4, pp. 735-742, 2003.

[6] C. Kravaris, V. Sotiropoulos, C. Georgiou, N. Kazantzis, M. Xiao, and A. J. Krener, "Nonlinear observer design for state and disturbance estimation," Systems $\mathcal{E}$ Control Letters, vol. 56, no. 11-12, pp. 730-735, 2007.

[7] B. Mahawan and Z.-H. Luo, "Repetitive control of tracking systems with time-varying periodic references," International Journal of Control, vol. 73, no. 1, pp. 1-10, 2000.

[8] C. L. Chen, G. T. C. Chiu, and J. Allebach, "Robust spatial-sampling controller design for banding reduction in electrophotographic process," Journal of Imaging Science and Technology, vol. 50, no. 6, pp. 530-536, 2006.

[9] C. L. Chen and G. T. C. Chiu, "Spatially periodic disturbance rejection with spatially sampled robust repetitive control," Journal of Dynamic Systems, Measurement, and Control, vol. 130, no. 2, pp. 11-21, 2008.

[10] M. Nakano, J. H. She, Y. Mastuo, and T. Hino, "Elimination of position-dependent disturbances in constant-speed-rotation control systems," Control Engineering Practice, vol. 4, no. 9, pp. 1241-1248, 1996.

[11] C. L. Chen and Y. H. Yang, "Spatially periodic disturbance rejection for uncertain rotational motion systems using spatial domain adaptive backstepping repetitive control," in Proceedings of the 33rd Annual Conference of the IEEE Industrial Electronics Society, pp. 638-643, Taipei, Taiwan, November 2007.

[12] Y. H. Yang and C. L. Chen, "Spatially periodic disturbance rejection using spatial-based output feedback adaptive backstepping repetitive control," in Proceeding of the American Control Conference, pp. 4117-4122, 2008.

[13] G. Kreisselmeier, "Adaptive observers with exponential rate of convergence," IEEE Transactions on Automatic Control, vol. 22, no. 1, pp. 2-8, 1977.

[14] R. Marino, G. L. Santosuosso, and P. Tomei, "Robust adaptive observers for nonlinear systems with bounded disturbances," IEEE Transactions on Automatic Control, vol. 46, no. 6, pp. 967-972, 2001.

[15] S. C. Tong, X. L. He, and H. G. Zhang, "A combined backstepping and small-gain approach to robust adaptive fuzzy output feedback control," IEEE Transactions on Fuzzy Systems, vol. 17, no. 5, pp. 1059$1069,2009$.

[16] S. Tong and Y. Li, "Observer-based fuzzy adaptive control for strict-feedback nonlinear systems," Fuzzy Sets and Systems, vol. 160, no. 12, pp. 1749-1764, 2009.

[17] S. Tong, C. Li, and Y. Li, "Fuzzy adaptive observer backstepping control for MIMO nonlinear systems," Fuzzy Sets and Systems, vol. 160, no. 19, pp. 2755-2775, 2009. 


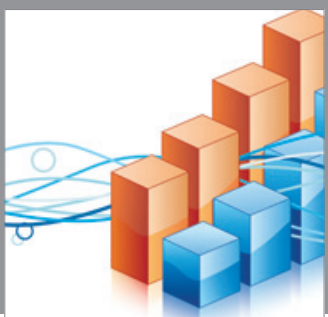

Advances in

Operations Research

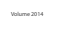

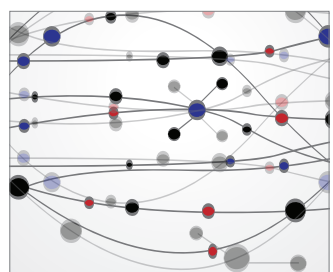

\section{The Scientific} World Journal
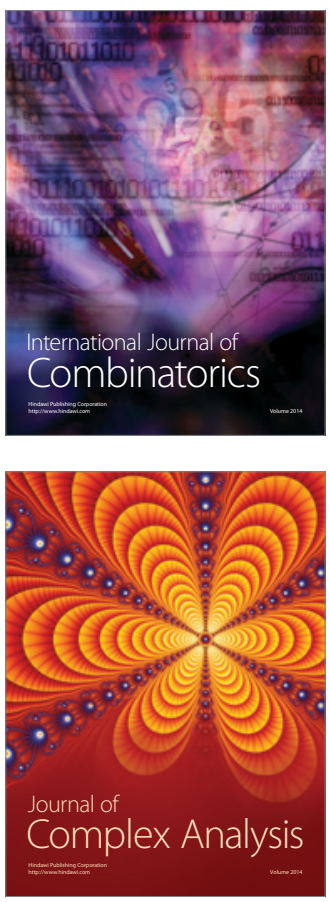

International Journal of

Mathematics and

Mathematical

Sciences
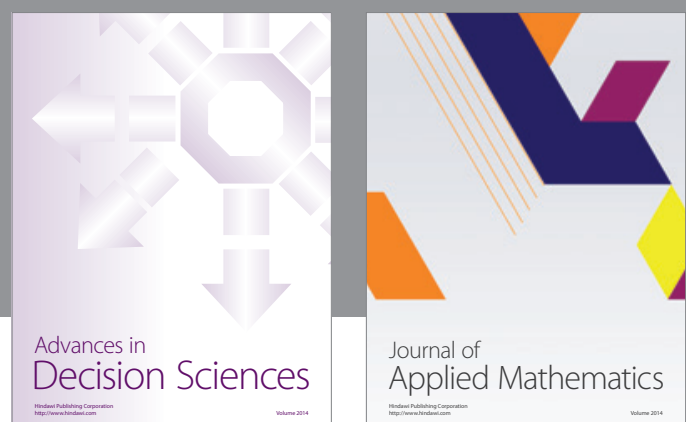

Journal of

Applied Mathematics
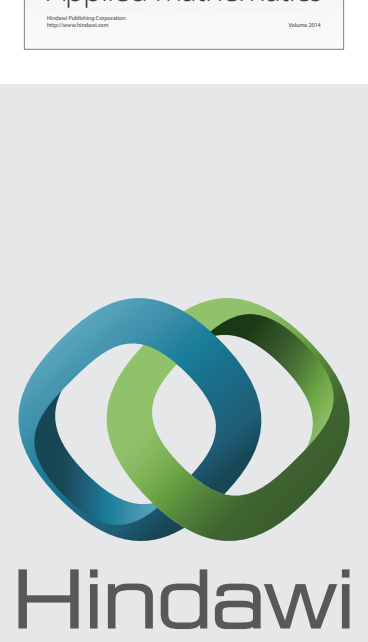

Submit your manuscripts at http://www.hindawi.com
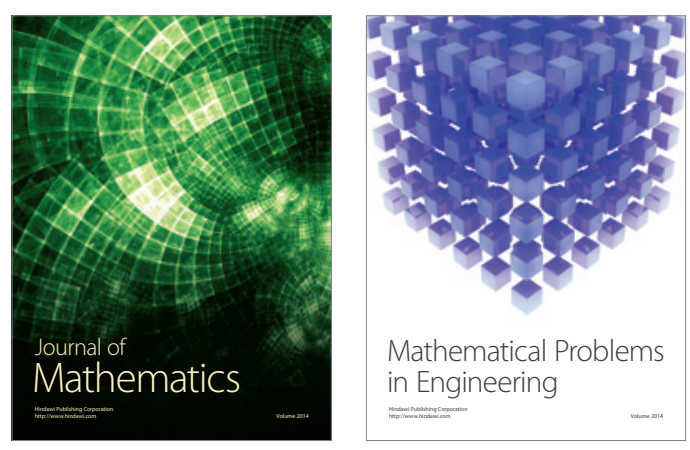

Mathematical Problems in Engineering
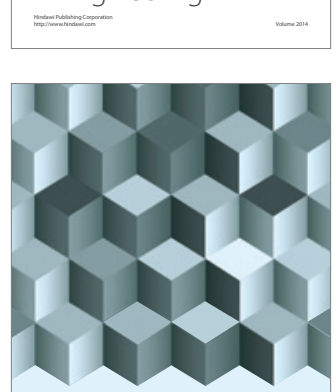

Journal of

Function Spaces
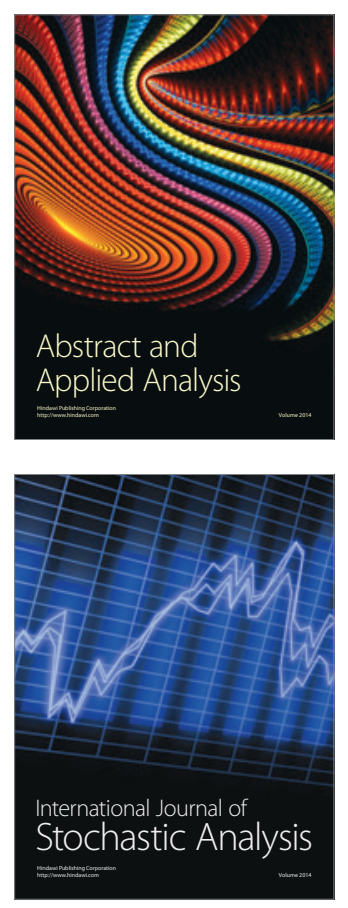

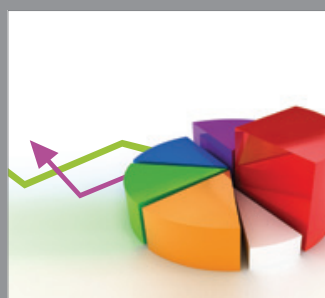

ournal of

Probability and Statistics

Promensencen
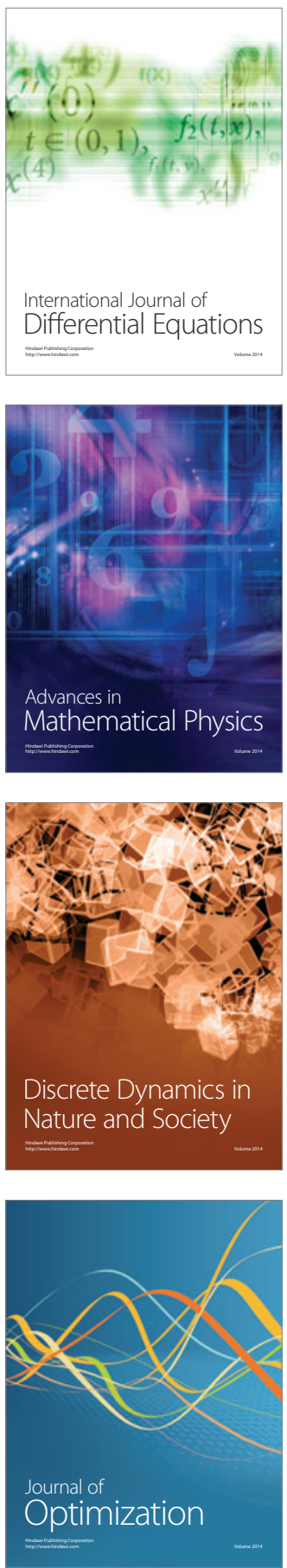